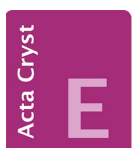

CRYSTALLOGRAPHIC COMMUNICATIONS

ISSN 2056-9890

Received 30 November 2015

Accepted 10 December 2015

Edited by J. Simpson, University of Otago, New Zealand

Keywords: crystal structure; amide derivative; pyridine; hydrogen bonding; $\pi-\pi$ contacts

CCDC reference: 1441555

Supporting information: this article has supporting information at journals.iucr.org/e

\section{Crystal structure of $\mathrm{N}$-\{4-[(6-chloropyridin-3-yl)- methoxy]phenyl\}-2,6-difluorobenzamide}

\author{
Ying Liang, ${ }^{*}$ Li-Qiao Shi and Zi-Wen Yang
}

Hubei Biopesticide Engineering Research Center, Hubei Academy of Agricultural Science, Wuhan 430064, People's Republic of China. *Correspondence e-mail: ying.liang@nberc.com

In the title compound, $\mathrm{C}_{19} \mathrm{H}_{13} \mathrm{ClF}_{2} \mathrm{~N}_{2} \mathrm{O}_{2}$, the conformation of the $\mathrm{N}-\mathrm{H}$ bond in the amide segment is anti to the $\mathrm{C}=\mathrm{O}$ bond. The molecule is not planar, with dihedral angles between the central benzene ring and the outer benzene and pyridyl rings of 73.35 (7) and $81.26(6)^{\circ}$, respectively. A weak intramolecular C$\mathrm{H} \cdots \mathrm{O}$ hydrogen bond occurs. In the crystal, $\mathrm{N}-\mathrm{H} \cdots \mathrm{N}, \mathrm{C}-\mathrm{H} \cdots \mathrm{O}$ and $\mathrm{C}-$ $\mathrm{H} \cdot \mathrm{F}$ hydrogen bonds lead to the formation of dimers. The $\mathrm{N}-\mathrm{H} \cdots \mathrm{N}$ inversion dimers are linked by $\pi-\pi$ contacts between adjacent pyridine rings [centroidcentroid $=3.8541(12) \AA]$ and $\mathrm{C}-\mathrm{H} \cdots \pi$ interactions. These contacts combine to stack the molecules along the $a$ axis.

\section{Chemical context}

Amide derivatives show diverse biological properties, acting as insecticides (Liu et al., 2004a), fungicides (Liu et al., 2004b) and acaricides (Shiga et al., 2003). Amides in regular commercial use include benzamide (flutolanil, fluopicolide), nicotinamide (boscalid) and thiazole carboxamide (thifluzamide, ethaboxam). As a part of our work on the synthesis of novel fluorine-containing compounds with good biological activities, we report herein on the crystal structure of the title compound,(I), Fig. 1.<smiles>O=C(Nc1ccc(OCc2ccc(Cl)nc2)cc1)c1c(F)cccc1F</smiles>

\section{Structural commentary}

The conformation of the $\mathrm{N}-\mathrm{H}$ and the $\mathrm{C}=\mathrm{O}$ bonds in the amide segment are anti to one another, similar to the

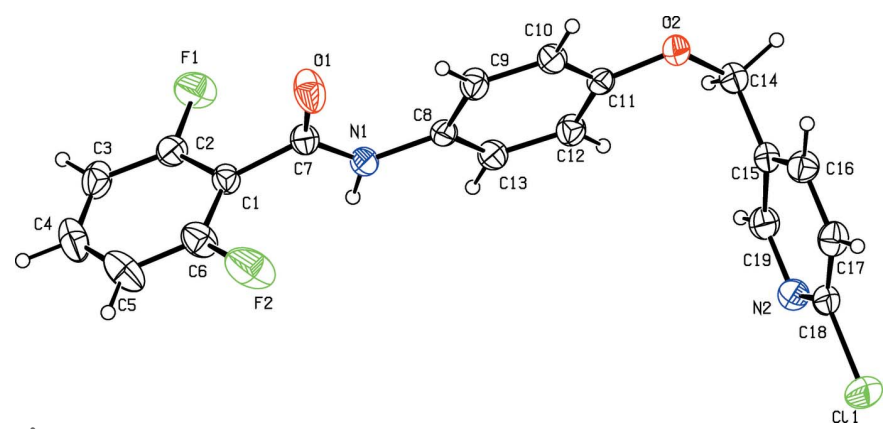

Figure 1

The structure of (I), showing $50 \%$ probability displacement ellipsoids and the atom-numbering scheme. 
Table 1

Hydrogen-bond geometry $\left(\AA,^{\circ}\right)$.

$\mathrm{Cg} 2$ is the centroid of the $\mathrm{C} 1-\mathrm{C} 6$ benzene ring.

\begin{tabular}{lllll}
\hline$D-\mathrm{H} \cdots A$ & $D-\mathrm{H}$ & $\mathrm{H} \cdots A$ & $D \cdots A$ & $D-\mathrm{H} \cdots A$ \\
\hline $\mathrm{C} 9-\mathrm{H} 9 \cdots \mathrm{O} 1$ & 0.93 & 2.27 & $2.863(2)$ & 121 \\
$\mathrm{~N} 1-\mathrm{H} 1 \cdots \mathrm{N} 2^{\mathrm{i}}$ & $0.88(2)$ & $2.24(2)$ & $3.109(2)$ & $170.6(18)$ \\
$\mathrm{C} 19-\mathrm{H} 19 \cdots \mathrm{F}^{\mathrm{ii}}$ & 0.93 & 2.54 & $3.309(2)$ & 140 \\
$\mathrm{C} 14-\mathrm{H} 14 B \cdots \mathrm{O} 1^{\mathrm{ii}}$ & 0.97 & 2.42 & $3.344(3)$ & 160 \\
$\mathrm{C} 16-\mathrm{H} 16 \cdots \mathrm{Cg} 2^{\mathrm{iii}}$ & 0.93 & 2.99 & $3.912(2)$ & 173 \\
\hline
\end{tabular}

Symmetry codes: (i) $-x,-y+1,-z$; (ii) $x-1, y, z$; (iii) $x-1, y, z+1$.

conformation observed in another amide compound (Gowda et al., 2010). The dihedral angle between the two benzene rings is $73.35(6)^{\circ}$ while that between the central benzene ring and the chloro-substituted pyridine ring is 81.26 (6). The amide residue $\mathrm{C} 1 / \mathrm{N} 1 / \mathrm{C} 7 / \mathrm{O} 1$ lies close to the plane of the central benzene ring, making a dihedral angle of $8.73(6)^{\circ}$. A weak intramolecular C9-H9...O1 hydrogen bond (Table 1) contributes to the planarity of this part of the molecule.

\section{Supramolecular features}

In the crystal structure, pairs of classical $\mathrm{N} 1-\mathrm{H} 1 \cdots \mathrm{N} 2^{\mathrm{i}}$ hydrogen bonds, Table 1 , link the molecules into inversion dimers and generate $R_{2}^{2}(22)$ rings (Bernstein et al., 1995). $\mathrm{C} 14-\mathrm{H} 14 B \cdots \mathrm{O} 1^{\mathrm{ii}}$ and $\mathrm{C} 19-\mathrm{H} 19 \cdots \mathrm{F} 2^{\mathrm{ii}}$ hydrogen bonds also form dimers, which enclose an $R_{2}^{2}(10)$ ring motif, Fig. 2 . The $\mathrm{N}-\mathrm{H} \cdots \mathrm{N}$ dimers are linked into chains along the $c$-axis direction by $\pi-\pi$ stacking interactions between adjacent pyridyl rings $\left[C g 1 \cdots C g 1^{\text {iv }}=3.8541(12) \AA\right.$; symmetry code: (iv) $1-x, 1-y, 1-z]$ augmented by a weak $\mathrm{C} 16-$ $\mathrm{H} 16 \cdots C g 2$ contact $(\mathrm{Cg} 2$ is the centroid of the $\mathrm{C} 1-\mathrm{C} 6$ benzene ring), Table 1, Fig. 3. These contacts combine to stack the molecules along the $a$ axis, Fig. 4.

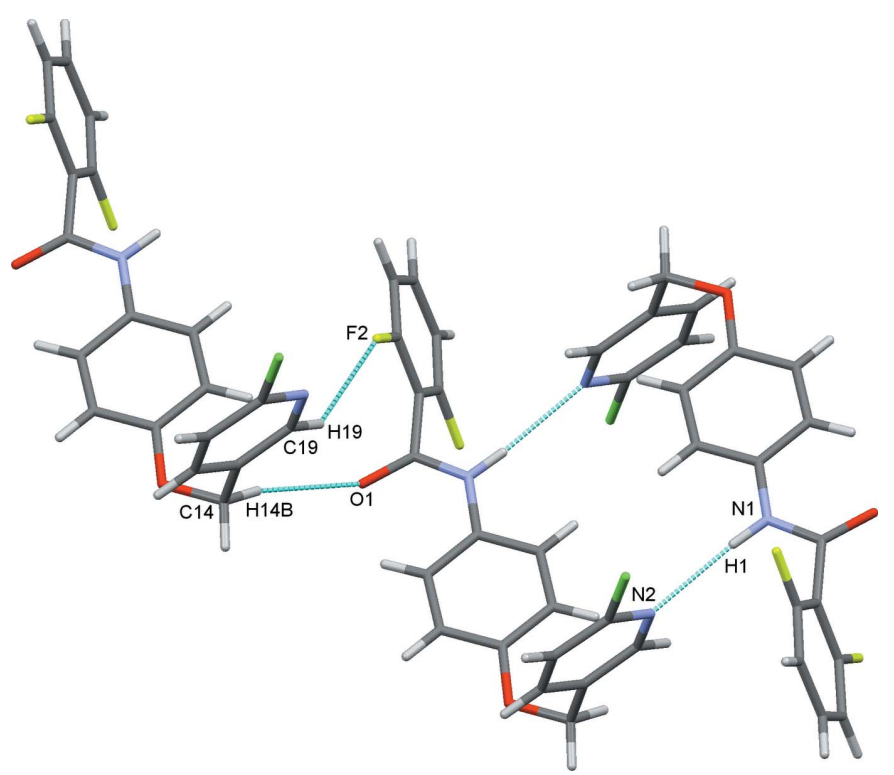

Figure 2

A pair of dimers with hydrogen bonds drawn as blue dashed lines.

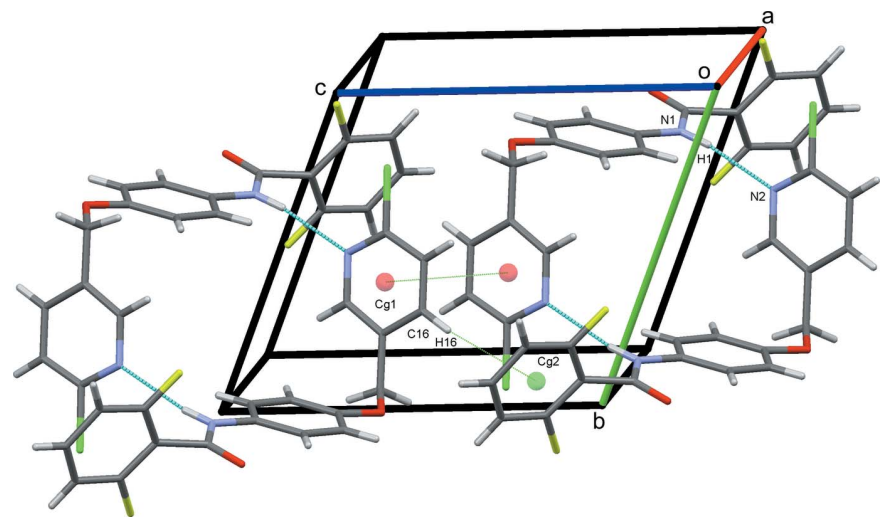

Figure 3

Chains of inversion dimers along the $c$-axis direction. Hydrogen bonds are drawn as dashed lines with $\pi-\pi$ and $\mathrm{C}-\mathrm{H} \cdots \pi$ contacts shown as green dotted lines.

\section{Synthesis and crystallization}

Triethylamine $(6 \mathrm{mmol})$ was added dropwise to a stirred solution of 4-(6-chloropyridin-3-yl) methoxy aniline $(5 \mathrm{mmol})$ and 2,6-difluorobenzoyl chloride $(5 \mathrm{mmol})$ in dry dichloromethane $(20 \mathrm{ml})$ at $275-277 \mathrm{~K}$. The mixture was stirred at $283-$ $288 \mathrm{~K}$ for $2 \mathrm{~h}$, then washed with $0.5 \%$ hydrochloric acid solution, and a saturated aqueous solution of sodium hydrogen carbonate, dried and evaporated. The residue was recrystallized from dichloromethane, giving colourless blocks of the title compound after three weeks.

\section{Database survey}

A search of the Cambridge Structural Database (Version 5.36 with three updates) (Groom \& Allen, 2014) for $N$-(4-(pyridin3-ylmethoxy)phenyl)benzamide or its substituted derivatives gave no hits. However, structures of eight substituted 2,6-difluoro- $N$-phenylbenzamide derivatives were found, see for example Cockroft et al. (2007); Spitaleri et al. (2004); Fun et al. (2010). Two structures of purely organic 3-(phenoxymethyl)pyridine derivatives have also been reported (Lakshminarayana et al., 2009; Liu et al., 2010) together with that of a cadmium complex of 4-[(6-chloropyridin-3-yl)methoxy]benzoate, Li et al. (2007).

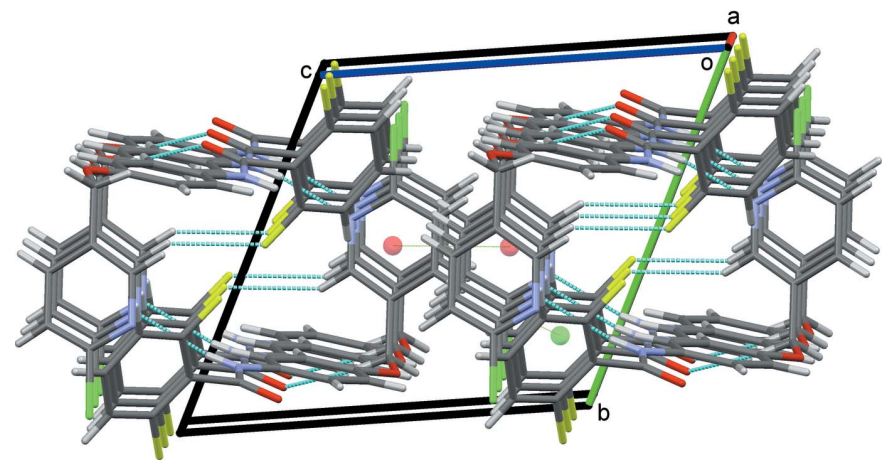

Figure 4

The overall packing for (I) viewed along the $a$-axis direction. 
Table 2

Experimental details.

\begin{tabular}{|c|c|}
\hline \multicolumn{2}{|l|}{ Crystal data } \\
\hline Chemical formula & $\mathrm{C}_{19} \mathrm{H}_{13} \mathrm{ClF}_{2} \mathrm{~N}_{2} \mathrm{O}_{2}$ \\
\hline$M_{\mathrm{r}}$ & 374.76 \\
\hline Crystal system, space group & Triclinic, $P \overline{1}$ \\
\hline Temperature (K) & 298 \\
\hline$a, b, c(\AA)$ & $\begin{array}{l}8.8173(11), 10.7036(13), \\
\quad 10.8452(14)\end{array}$ \\
\hline$\alpha, \beta, \gamma\left({ }^{\circ}\right)$ & $61.939(2), 77.597$ (2), $69.636(2)$ \\
\hline$V\left(\AA^{3}\right)$ & $845.15(18)$ \\
\hline$Z$ & 2 \\
\hline Radiation type & Мo $K \alpha$ \\
\hline$\mu\left(\mathrm{mm}^{-1}\right)$ & 0.26 \\
\hline Crystal size $(\mathrm{mm})$ & $0.16 \times 0.12 \times 0.10$ \\
\hline \multicolumn{2}{|l|}{ Data collection } \\
\hline Diffractometer & $\begin{array}{l}\text { Bruker SMART APEX CCD area } \\
\text { detector }\end{array}$ \\
\hline Absorption correction & $\begin{array}{l}\text { Multi-scan (SADABS; Sheldrick, } \\
\text { 2001) }\end{array}$ \\
\hline$T_{\min }, T_{\max }$ & $0.959,0.974$ \\
\hline $\begin{array}{l}\text { No. of measured, independent and } \\
\text { observed }[I>2 \sigma(I)] \text { reflections }\end{array}$ & $5474,3271,2886$ \\
\hline$R_{\text {int }}$ & 0.029 \\
\hline$(\sin \theta / \lambda)_{\max }\left(\AA^{-1}\right)$ & 0.617 \\
\hline \multicolumn{2}{|l|}{ Refinement } \\
\hline$R\left[F^{2}>2 \sigma\left(F^{2}\right)\right], w R\left(F^{2}\right), S$ & $0.045,0.116,1.06$ \\
\hline No. of reflections & 3271 \\
\hline No. of parameters & 239 \\
\hline $\mathrm{H}$-atom treatment & $\begin{array}{l}\mathrm{H} \text { atoms treated by a mixture of } \\
\text { independent and constrained } \\
\text { refinement }\end{array}$ \\
\hline$\Delta \rho_{\max }, \Delta \rho_{\min }\left(\mathrm{e} \AA^{-3}\right)$ & $0.21,-0.23$ \\
\hline
\end{tabular}

Computer programs: SMART and SAINT (Bruker, 2000), SHELXS97, SHELXL97 and SHELXTL (Sheldrick, 2008) and Mercury (Macrae et al., 2008).

\section{Refinement}

Crystal data, data collection and structure refinement details are summarized in Table 2. The $\mathrm{NH} \mathrm{H}$ atom was located in a difference Fourier map and freely refined. The C-bound $\mathrm{H}$ atoms were positioned geometrically and refined using a riding model with $d(\mathrm{C}-\mathrm{H})=0.93-0.97 \AA$ and $U_{\text {iso }}(\mathrm{H})=$ $1.2 U_{\text {eq }}(\mathrm{C})$.

\section{Acknowledgements}

We gratefully acknowledge the financial support of this work by the Youth Science Foundation of Hubei Academy of Agricultural Sciences (grant No. 2013NKYJJ22) and the Key Laboratory of Integrated Pest Management in Crops in Central China, the Ministry of Agriculture and Hubei Key Laboratory of Crop Diseases, Insect Pests and Weed Control (grant No. 2015ZTSJJ9).

\section{References}

Bernstein, J., Davis, R. E., Shimoni, L. \& Chang, N.-L. (1995). Angew. Chem. Int. Ed. Engl. 34, 1555-1573.

Bruker (2000). SMART, SAINT and SADABS. Bruker AXS Inc., Madison, Wisconsin, USA.

Cockroft, S. L., Perkins, J., Zonta, C., Adams, H., Spey, S. E., Low, C. M. R., Vinter, J. G., Lawson, K. R., Urch, C. J. \& Hunter, C. A. (2007). Org. Biomol. Chem. 5, 1062-1080.

Fun, H.-K., Goh, J. H., Gowda, J., Khader, A. M. \& Kalluraya, B. (2010). Acta Cryst. E66, o3192.

Gowda, B. T., Tokarčík, M., Shakuntala, K., Kožíšek, J. \& Fuess, H. (2010). Acta Cryst. E66, o1529-o1530.

Groom, C. R. \& Allen, F. H. (2014). Angew. Chem. Int. Ed. 53, 662671.

Lakshminarayana, B. N., Prasad, J. S., Venu, T. D., Manuprasad, B. K., Sridhar, M. A. \& Shashikanth, S. (2009). Mol. Cryst. Liq. Cryst. 515, 207-214.

Li, S.-L., Liu, J. \& Liu, Y.-Y. (2007). Acta Cryst. E63, m2956.

Liu, C. L., Li, L. \& Li, Z. M. (2004a). Bioorg. Med. Chem. 12, $2825-$ 2830.

Liu, C. L., Li, Z. M. \& Zhong, B. (2004b). J. Fluor. Chem. 125, $1287-$ 1290.

Liu, X.-H., Liu, H.-F., Shen, X., Song, B.-A., Bhadury, P. S., Zhu, H.-L., Liu, J.-X. \& Qi, X.-B. (2010). Bioorg. Med. Chem. Lett. 20, 4163-4167.

Macrae, C. F., Bruno, I. J., Chisholm, J. A., Edgington, P. R., McCabe, P., Pidcock, E., Rodriguez-Monge, L., Taylor, R., van de Streek, J. \& Wood, P. A. (2008). J. Appl. Cryst. 41, 466-470.

Sheldrick, G. M. (2001). SADABS. University of Göttingen, Germany.

Sheldrick, G. M. (2008). Acta Cryst. A64, 112-122.

Shiga, Y., Okada, I. \& Fukuchi, T. (2003). J. Pestic. Sci. 28, 310-312.

Spitaleri, A., Hunter, C. A., McCabe, J. F., Packer, M. J. \& Cockroft, S. L. (2004). CrystEngComm, 6, 490-493. 


\section{supporting information}

Acta Cryst. (2016). E72, 60-62 [doi:10.1107/S2056989015023701]

Crystal structure of N-\{4-[(6-chloropyridin-3-yl)methoxy]phenyl\}-2,6-difluorobenzamide

\section{Ying Liang, Li-Qiao Shi and Zi-Wen Yang}

Computing details

Data collection: SMART (Bruker, 2000); cell refinement: SAINT (Bruker, 2000); data reduction: SAINT (Bruker, 2000); program(s) used to solve structure: SHELXS97 (Sheldrick, 2008); program(s) used to refine structure: SHELXL97 (Sheldrick, 2008); molecular graphics: SHELXTL (Sheldrick, 2008) and Mercury (Macrae et al., 2008); software used to prepare material for publication: SHELXTL (Sheldrick, 2008).

N-\{4-[(6-Chloropyridin-3-yl)methoxy]phenyl\}-2,6-difluorobenzamide

\section{Crystal data}

$\mathrm{C}_{19} \mathrm{H}_{13} \mathrm{ClF}_{2} \mathrm{~N}_{2} \mathrm{O}_{2}$

$M_{r}=374.76$

Triclinic, $P \overline{1}$

Hall symbol: -P 1

$a=8.8173(11) \AA$

$b=10.7036(13) \AA$

$c=10.8452(14) \AA$

$\alpha=61.939(2)^{\circ}$

$\beta=77.597(2)^{\circ}$

$\gamma=69.636(2)^{\circ}$

$V=845.15(18) \AA^{3}$

\section{Data collection}

Bruker SMART APEX CCD area-detector diffractometer

Radiation source: fine-focus sealed tube

Graphite monochromator

$\varphi$ and $\omega$ scans

Absorption correction: multi-scan

(SADABS; Sheldrick, 2001)

$T_{\min }=0.959, T_{\max }=0.974$

\section{Refinement}

Refinement on $F^{2}$

Least-squares matrix: full

$R\left[F^{2}>2 \sigma\left(F^{2}\right)\right]=0.045$

$w R\left(F^{2}\right)=0.116$

$S=1.06$

3271 reflections

239 parameters

0 restraints

$$
\begin{aligned}
& Z=2 \\
& F(000)=384 \\
& D_{\mathrm{x}}=1.473 \mathrm{Mg} \mathrm{m}^{-3}
\end{aligned}
$$

Mo $K \alpha$ radiation, $\lambda=0.71073 \AA$

Cell parameters from 2810 reflections

$\theta=2.3-28.3^{\circ}$

$\mu=0.26 \mathrm{~mm}^{-1}$

$T=298 \mathrm{~K}$

Block, colorless

$0.16 \times 0.12 \times 0.10 \mathrm{~mm}$

5474 measured reflections

3271 independent reflections

2886 reflections with $I>2 \sigma(I)$

$R_{\text {int }}=0.029$

$\theta_{\text {max }}=26.0^{\circ}, \theta_{\min }=2.1^{\circ}$

$h=-10 \rightarrow 10$

$k=-13 \rightarrow 13$

$l=-12 \rightarrow 13$

Primary atom site location: structure-invariant direct methods

Secondary atom site location: difference Fourier map

Hydrogen site location: inferred from neighbouring sites

$\mathrm{H}$ atoms treated by a mixture of independent and constrained refinement 
$w=1 /\left[\sigma^{2}\left(F_{\mathrm{o}}^{2}\right)+(0.0526 P)^{2}+0.196 P\right]$

where $P=\left(F_{\mathrm{o}}^{2}+2 F_{\mathrm{c}}{ }^{2}\right) / 3$

$(\Delta / \sigma)_{\max }<0.001$

$$
\Delta \rho_{\max }=0.21 \text { e } \AA^{-3}
$$

\section{Special details}

Geometry. All esds (except the esd in the dihedral angle between two 1.s. planes) are estimated using the full covariance matrix. The cell esds are taken into account individually in the estimation of esds in distances, angles and torsion angles; correlations between esds in cell parameters are only used when they are defined by crystal symmetry. An approximate (isotropic) treatment of cell esds is used for estimating esds involving l.s. planes.

Refinement. Refinement of $\mathrm{F}^{2}$ against ALL reflections. The weighted R-factor $\mathrm{wR}$ and goodness of fit $\mathrm{S}$ are based on $\mathrm{F}^{2}$, conventional R-factors $R$ are based on $F$, with $F$ set to zero for negative $\mathrm{F}^{2}$. The threshold expression of $\mathrm{F}^{2}>2 \operatorname{sigma}\left(\mathrm{F}^{2}\right)$ is used only for calculating R-factors(gt) etc. and is not relevant to the choice of reflections for refinement. R-factors based on $\mathrm{F}^{2}$ are statistically about twice as large as those based on F, and R- factors based on ALL data will be even larger.

Fractional atomic coordinates and isotropic or equivalent isotropic displacement parameters $\left(\AA^{2}\right)$

\begin{tabular}{|c|c|c|c|c|}
\hline & $x$ & $y$ & $z$ & $U_{\text {iso }} * / U_{\text {eq }}$ \\
\hline $\mathrm{C} 1$ & $0.7630(2)$ & $0.2167(2)$ & $-0.02643(18)$ & $0.0427(4)$ \\
\hline $\mathrm{C} 2$ & $0.8270(2)$ & $0.1167(2)$ & -0.08394 (19) & $0.0478(4)$ \\
\hline $\mathrm{C} 3$ & $0.9422(3)$ & $0.1317(3)$ & $-0.1918(2)$ & $0.0611(6)$ \\
\hline $\mathrm{H} 3$ & 0.9816 & 0.0618 & -0.2276 & $0.073 *$ \\
\hline $\mathrm{C} 4$ & $0.9982(3)$ & $0.2526(3)$ & $-0.2461(2)$ & $0.0706(7)$ \\
\hline $\mathrm{H} 4$ & 1.0773 & 0.2644 & -0.3195 & $0.085^{*}$ \\
\hline $\mathrm{C} 5$ & $0.9403(3)$ & $0.3566(3)$ & $-0.1949(2)$ & $0.0750(7)$ \\
\hline H5 & 0.9787 & 0.4388 & -0.2327 & $0.090^{*}$ \\
\hline C6 & $0.8239(3)$ & $0.3360(2)$ & $-0.0861(2)$ & $0.0602(5)$ \\
\hline $\mathrm{C} 7$ & $0.6521(2)$ & $0.1886(2)$ & $0.10413(18)$ & $0.0432(4)$ \\
\hline C8 & $0.3644(2)$ & $0.21024(17)$ & $0.18987(17)$ & $0.0391(4)$ \\
\hline C9 & $0.3813(2)$ & $0.1688(2)$ & $0.32952(18)$ & $0.0462(4)$ \\
\hline H9 & 0.4817 & 0.1503 & 0.3590 & $0.055^{*}$ \\
\hline $\mathrm{C} 10$ & $0.2489(2)$ & $0.1551(2)$ & $0.42425(18)$ & $0.0461(4)$ \\
\hline $\mathrm{H} 10$ & 0.2609 & 0.1273 & 0.5176 & $0.055^{*}$ \\
\hline $\mathrm{C} 11$ & $0.0990(2)$ & $0.18196(17)$ & $0.38294(18)$ & $0.0408(4)$ \\
\hline $\mathrm{C} 12$ & $0.0809(2)$ & $0.2233(2)$ & $0.24416(19)$ & $0.0470(4)$ \\
\hline H12 & -0.0198 & 0.2422 & 0.2149 & $0.056^{*}$ \\
\hline $\mathrm{C} 13$ & $0.2140(2)$ & $0.2363(2)$ & $0.14935(18)$ & $0.0461(4)$ \\
\hline $\mathrm{H} 13$ & 0.2019 & 0.2631 & 0.0563 & $0.055^{*}$ \\
\hline $\mathrm{C} 14$ & $-0.1851(2)$ & $0.2030(2)$ & $0.4525(2)$ & $0.0497(5)$ \\
\hline H14A & -0.2488 & 0.1521 & 0.5341 & $0.060^{*}$ \\
\hline H14B & -0.1861 & 0.1737 & 0.3806 & $0.060^{*}$ \\
\hline $\mathrm{C} 15$ & $-0.2607(2)$ & $0.36755(19)$ & $0.40005(18)$ & $0.0414(4)$ \\
\hline $\mathrm{C} 16$ & $-0.2680(2)$ & $0.4370(2)$ & $0.4829(2)$ & $0.0498(5)$ \\
\hline H16 & -0.2264 & 0.3819 & 0.5710 & $0.060^{*}$ \\
\hline $\mathrm{C} 17$ & $-0.3362(2)$ & $0.5864(2)$ & $0.4347(2)$ & $0.0506(5)$ \\
\hline H17 & -0.3418 & 0.6348 & 0.4885 & $0.061^{*}$ \\
\hline $\mathrm{C} 18$ & $-0.3961(2)$ & $0.6622(2)$ & $0.3043(2)$ & $0.0479(4)$ \\
\hline C19 & $-0.3260(2)$ & $0.4551(2)$ & $0.2721(2)$ & $0.0503(5)$ \\
\hline H19 & -0.3230 & 0.4098 & 0.2161 & $0.060^{*}$ \\
\hline $\mathrm{Cl} 1$ & $-0.48341(8)$ & $0.85162(6)$ & $0.23999(7)$ & $0.0783(2)$ \\
\hline
\end{tabular}




$\begin{array}{lllll}\text { F1 } & 0.77345(18) & -0.00346(13) & -0.02627(13) & 0.0741(4) \\ \text { F2 } & 0.7661(2) & 0.43493(16) & -0.03160(16) & 0.1031(6) \\ \text { N1 } & 0.49454(18) & 0.22686(16) & 0.08547(16) & 0.0430(4) \\ \text { H1 } & 0.468(2) & 0.265(2) & -0.001(2) & 0.052^{*} \\ \text { N2 } & -0.3942(2) & 0.60203(18) & 0.22226(16) & 0.0537(4) \\ \text { O1 } & 0.71012(17) & 0.1345(2) & 0.21658(15) & 0.0727(5) \\ \text { O2 } & -0.02172(15) & 0.16015(14) & 0.48849(13) & 0.0495(3)\end{array}$

Atomic displacement parameters $\left(\AA^{2}\right)$

\begin{tabular}{lllllll}
\hline & $U^{11}$ & $U^{22}$ & $U^{33}$ & $U^{12}$ & $U^{13}$ & $U^{23}$ \\
\hline C1 & $0.0410(9)$ & $0.0482(10)$ & $0.0375(9)$ & $-0.0112(8)$ & $-0.0013(7)$ & $-0.0189(8)$ \\
C2 & $0.0514(11)$ & $0.0483(10)$ & $0.0415(10)$ & $-0.0084(8)$ & $-0.0054(8)$ & $-0.0207(8)$ \\
C3 & $0.0524(12)$ & $0.0763(15)$ & $0.0463(11)$ & $0.0010(11)$ & $-0.0033(9)$ & $-0.0335(11)$ \\
C4 & $0.0484(12)$ & $0.116(2)$ & $0.0456(12)$ & $-0.0288(13)$ & $0.0066(9)$ & $-0.0342(13)$ \\
C5 & $0.0860(17)$ & $0.1005(19)$ & $0.0517(13)$ & $-0.0615(15)$ & $0.0073(12)$ & $-0.0240(13)$ \\
C6 & $0.0785(15)$ & $0.0630(13)$ & $0.0489(11)$ & $-0.0310(11)$ & $0.0043(10)$ & $-0.0278(10)$ \\
C7 & $0.0470(10)$ & $0.0465(9)$ & $0.0385(9)$ & $-0.0125(8)$ & $-0.0001(8)$ & $-0.0220(8)$ \\
C8 & $0.0434(9)$ & $0.0330(8)$ & $0.0364(9)$ & $-0.0069(7)$ & $0.0014(7)$ & $-0.0159(7)$ \\
C9 & $0.0425(10)$ & $0.0540(10)$ & $0.0408(10)$ & $-0.0085(8)$ & $-0.0032(8)$ & $-0.0230(8)$ \\
C10 & $0.0508(11)$ & $0.0483(10)$ & $0.0347(9)$ & $-0.0076(8)$ & $-0.0026(8)$ & $-0.0188(8)$ \\
C11 & $0.0453(10)$ & $0.0322(8)$ & $0.0391(9)$ & $-0.0062(7)$ & $0.0016(7)$ & $-0.0160(7)$ \\
C12 & $0.0423(10)$ & $0.0523(10)$ & $0.0444(10)$ & $-0.0092(8)$ & $-0.0041(8)$ & $-0.0219(9)$ \\
C13 & $0.0512(11)$ & $0.0480(10)$ & $0.0355(9)$ & $-0.0090(8)$ & $-0.0030(8)$ & $-0.0188(8)$ \\
C14 & $0.0457(10)$ & $0.0462(10)$ & $0.0515(11)$ & $-0.0141(8)$ & $0.0055(8)$ & $-0.0194(9)$ \\
C15 & $0.0353(9)$ & $0.0447(9)$ & $0.0424(9)$ & $-0.0120(7)$ & $0.0045(7)$ & $-0.0198(8)$ \\
C16 & $0.0479(11)$ & $0.0540(11)$ & $0.0457(10)$ & $-0.0101(8)$ & $-0.0079(8)$ & $-0.0214(9)$ \\
C17 & $0.0483(11)$ & $0.0552(11)$ & $0.0573(12)$ & $-0.0160(9)$ & $0.0002(9)$ & $-0.0321(10)$ \\
C18 & $0.0379(9)$ & $0.0427(9)$ & $0.0550(11)$ & $-0.0123(7)$ & $0.0062(8)$ & $-0.0179(9)$ \\
C19 & $0.0544(11)$ & $0.0524(11)$ & $0.0444(10)$ & $-0.0118(9)$ & $0.0011(8)$ & $-0.0254(9)$ \\
C11 & $0.0732(4)$ & $0.0415(3)$ & $0.0948(5)$ & $-0.0080(2)$ & $0.0032(3)$ & $-0.0186(3)$ \\
F1 & $0.1111(11)$ & $0.0569(7)$ & $0.0632(8)$ & $-0.0301(7)$ & $0.0031(7)$ & $-0.0322(6)$ \\
F2 & $0.1807(18)$ & $0.0716(9)$ & $0.0786(10)$ & $-0.0650(11)$ & $0.0283(10)$ & $-0.0444(8)$ \\
N1 & $0.0453(9)$ & $0.0451(8)$ & $0.0327(8)$ & $-0.0082(6)$ & $-0.0008(6)$ & $-0.0161(7)$ \\
N2 & $0.0541(10)$ & $0.0527(9)$ & $0.0428(9)$ & $-0.0079(7)$ & $-0.0019(7)$ & $-0.0173(7)$ \\
O1 & $0.0500(8)$ & $0.1190(14)$ & $0.0444(8)$ & $-0.0210(9)$ & $-0.0008(7)$ & $-0.0348(9)$ \\
O2 & $0.0436(7)$ & $0.0507(7)$ & $0.0411(7)$ & $-0.0063(6)$ & $0.0034(5)$ & $-0.0170(6)$ \\
& & & & & & \\
& & & & & &
\end{tabular}

Geometric parameters $\left(\AA,{ }^{\circ}\right)$

\begin{tabular}{llll}
\hline $\mathrm{C} 1-\mathrm{C} 6$ & $1.374(3)$ & $\mathrm{C} 11-\mathrm{O} 2$ & $1.377(2)$ \\
$\mathrm{C} 1-\mathrm{C} 2$ & $1.382(2)$ & $\mathrm{C} 11-\mathrm{C} 12$ & $1.383(2)$ \\
$\mathrm{C} 1-\mathrm{C} 7$ & $1.504(2)$ & $\mathrm{C} 12-\mathrm{C} 13$ & $1.384(2)$ \\
$\mathrm{C} 2-\mathrm{F} 1$ & $1.344(2)$ & $\mathrm{C} 12-\mathrm{H} 12$ & 0.9300 \\
$\mathrm{C} 2-\mathrm{C} 3$ & $1.361(3)$ & $\mathrm{C} 13-\mathrm{H} 13$ & 0.9300 \\
$\mathrm{C} 3-\mathrm{C} 4$ & $1.366(3)$ & $\mathrm{C} 14-\mathrm{O} 2$ & $1.432(2)$ \\
$\mathrm{C} 3-\mathrm{H} 3$ & 0.9300 & $\mathrm{C} 14-\mathrm{C} 15$ & $1.508(2)$ \\
$\mathrm{C} 4-\mathrm{C} 5$ & $1.368(4)$ & $\mathrm{C} 14-\mathrm{H} 14 \mathrm{~A}$ & 0.9700
\end{tabular}




\begin{tabular}{|c|c|c|c|}
\hline $\mathrm{C} 4-\mathrm{H} 4$ & 0.9300 & C14-H14B & 0.9700 \\
\hline $\mathrm{C} 5-\mathrm{C} 6$ & $1.374(3)$ & $\mathrm{C} 15-\mathrm{C} 19$ & $1.371(3)$ \\
\hline $\mathrm{C} 5-\mathrm{H} 5$ & 0.9300 & $\mathrm{C} 15-\mathrm{C} 16$ & $1.391(2)$ \\
\hline $\mathrm{C} 6-\mathrm{F} 2$ & $1.347(2)$ & $\mathrm{C} 16-\mathrm{C} 17$ & $1.368(3)$ \\
\hline $\mathrm{C} 7-\mathrm{O} 1$ & $1.217(2)$ & $\mathrm{C} 16-\mathrm{H} 16$ & 0.9300 \\
\hline $\mathrm{C} 7-\mathrm{N} 1$ & $1.336(2)$ & $\mathrm{C} 17-\mathrm{C} 18$ & $1.372(3)$ \\
\hline $\mathrm{C} 8-\mathrm{C} 13$ & $1.381(3)$ & $\mathrm{C} 17-\mathrm{H} 17$ & 0.9300 \\
\hline $\mathrm{C} 8-\mathrm{C} 9$ & $1.390(2)$ & $\mathrm{C} 18-\mathrm{N} 2$ & $1.316(2)$ \\
\hline $\mathrm{C} 8-\mathrm{N} 1$ & $1.423(2)$ & $\mathrm{C} 18-\mathrm{Cl1}$ & $1.7328(19)$ \\
\hline $\mathrm{C} 9-\mathrm{C} 10$ & $1.379(2)$ & $\mathrm{C} 19-\mathrm{N} 2$ & $1.345(2)$ \\
\hline C9-H9 & 0.9300 & C19-H19 & 0.9300 \\
\hline $\mathrm{C} 10-\mathrm{C} 11$ & $1.378(3)$ & $\mathrm{N} 1-\mathrm{H} 1$ & $0.88(2)$ \\
\hline $\mathrm{C} 10-\mathrm{H} 10$ & 0.9300 & & \\
\hline $\mathrm{C} 6-\mathrm{C} 1-\mathrm{C} 2$ & $115.21(17)$ & $\mathrm{C} 11-\mathrm{C} 12-\mathrm{C} 13$ & $119.36(17)$ \\
\hline $\mathrm{C} 6-\mathrm{C} 1-\mathrm{C} 7$ & $121.35(16)$ & $\mathrm{C} 11-\mathrm{C} 12-\mathrm{H} 12$ & 120.3 \\
\hline $\mathrm{C} 2-\mathrm{C} 1-\mathrm{C} 7$ & $122.93(16)$ & $\mathrm{C} 13-\mathrm{C} 12-\mathrm{H} 12$ & 120.3 \\
\hline $\mathrm{F} 1-\mathrm{C} 2-\mathrm{C} 3$ & $119.06(18)$ & $\mathrm{C} 8-\mathrm{C} 13-\mathrm{C} 12$ & $121.47(16)$ \\
\hline $\mathrm{F} 1-\mathrm{C} 2-\mathrm{C} 1$ & $117.06(16)$ & $\mathrm{C} 8-\mathrm{C} 13-\mathrm{H} 13$ & 119.3 \\
\hline $\mathrm{C} 3-\mathrm{C} 2-\mathrm{C} 1$ & $123.86(19)$ & $\mathrm{C} 12-\mathrm{C} 13-\mathrm{H} 13$ & 119.3 \\
\hline $\mathrm{C} 2-\mathrm{C} 3-\mathrm{C} 4$ & $118.0(2)$ & $\mathrm{O} 2-\mathrm{C} 14-\mathrm{C} 15$ & $111.33(15)$ \\
\hline $\mathrm{C} 2-\mathrm{C} 3-\mathrm{H} 3$ & 121.0 & $\mathrm{O} 2-\mathrm{C} 14-\mathrm{H} 14 \mathrm{~A}$ & 109.4 \\
\hline $\mathrm{C} 4-\mathrm{C} 3-\mathrm{H} 3$ & 121.0 & $\mathrm{C} 15-\mathrm{C} 14-\mathrm{H} 14 \mathrm{~A}$ & 109.4 \\
\hline $\mathrm{C} 3-\mathrm{C} 4-\mathrm{C} 5$ & $121.5(2)$ & $\mathrm{O} 2-\mathrm{C} 14-\mathrm{H} 14 \mathrm{~B}$ & 109.4 \\
\hline $\mathrm{C} 3-\mathrm{C} 4-\mathrm{H} 4$ & 119.3 & $\mathrm{C} 15-\mathrm{C} 14-\mathrm{H} 14 \mathrm{~B}$ & 109.4 \\
\hline $\mathrm{C} 5-\mathrm{C} 4-\mathrm{H} 4$ & 119.3 & $\mathrm{H} 14 \mathrm{~A}-\mathrm{C} 14-\mathrm{H} 14 \mathrm{~B}$ & 108.0 \\
\hline $\mathrm{C} 4-\mathrm{C} 5-\mathrm{C} 6$ & $118.1(2)$ & $\mathrm{C} 19-\mathrm{C} 15-\mathrm{C} 16$ & $116.97(17)$ \\
\hline $\mathrm{C} 4-\mathrm{C} 5-\mathrm{H} 5$ & 121.0 & $\mathrm{C} 19-\mathrm{C} 15-\mathrm{C} 14$ & $122.83(17)$ \\
\hline $\mathrm{C} 6-\mathrm{C} 5-\mathrm{H} 5$ & 121.0 & $\mathrm{C} 16-\mathrm{C} 15-\mathrm{C} 14$ & $120.19(16)$ \\
\hline $\mathrm{F} 2-\mathrm{C} 6-\mathrm{C} 1$ & $116.98(18)$ & $\mathrm{C} 17-\mathrm{C} 16-\mathrm{C} 15$ & $119.99(17)$ \\
\hline $\mathrm{F} 2-\mathrm{C} 6-\mathrm{C} 5$ & $119.7(2)$ & $\mathrm{C} 17-\mathrm{C} 16-\mathrm{H} 16$ & 120.0 \\
\hline $\mathrm{C} 1-\mathrm{C} 6-\mathrm{C} 5$ & $123.4(2)$ & $\mathrm{C} 15-\mathrm{C} 16-\mathrm{H} 16$ & 120.0 \\
\hline $\mathrm{O} 1-\mathrm{C} 7-\mathrm{N} 1$ & $125.21(17)$ & $\mathrm{C} 16-\mathrm{C} 17-\mathrm{C} 18$ & $117.59(17)$ \\
\hline $\mathrm{O} 1-\mathrm{C} 7-\mathrm{C} 1$ & $118.93(16)$ & $\mathrm{C} 16-\mathrm{C} 17-\mathrm{H} 17$ & 121.2 \\
\hline $\mathrm{N} 1-\mathrm{C} 7-\mathrm{C} 1$ & $115.86(15)$ & $\mathrm{C} 18-\mathrm{C} 17-\mathrm{H} 17$ & 121.2 \\
\hline $\mathrm{C} 13-\mathrm{C} 8-\mathrm{C} 9$ & $118.73(16)$ & $\mathrm{N} 2-\mathrm{C} 18-\mathrm{C} 17$ & $124.97(17)$ \\
\hline $\mathrm{C} 13-\mathrm{C} 8-\mathrm{N} 1$ & $117.80(15)$ & $\mathrm{N} 2-\mathrm{C} 18-\mathrm{Cl} 1$ & $116.22(15)$ \\
\hline $\mathrm{C} 9-\mathrm{C} 8-\mathrm{N} 1$ & $123.47(16)$ & $\mathrm{C} 17-\mathrm{C} 18-\mathrm{Cl1}$ & $118.81(15)$ \\
\hline $\mathrm{C} 10-\mathrm{C} 9-\mathrm{C} 8$ & $119.84(17)$ & $\mathrm{N} 2-\mathrm{C} 19-\mathrm{C} 15$ & $124.31(17)$ \\
\hline $\mathrm{C} 10-\mathrm{C} 9-\mathrm{H} 9$ & 120.1 & $\mathrm{~N} 2-\mathrm{C} 19-\mathrm{H} 19$ & 117.8 \\
\hline $\mathrm{C} 8-\mathrm{C} 9-\mathrm{H} 9$ & 120.1 & $\mathrm{C} 15-\mathrm{C} 19-\mathrm{H} 19$ & 117.8 \\
\hline $\mathrm{C} 11-\mathrm{C} 10-\mathrm{C} 9$ & $121.11(16)$ & $\mathrm{C} 7-\mathrm{N} 1-\mathrm{C} 8$ & $127.66(15)$ \\
\hline $\mathrm{C} 11-\mathrm{C} 10-\mathrm{H} 10$ & 119.4 & $\mathrm{C} 7-\mathrm{N} 1-\mathrm{H} 1$ & $116.4(13)$ \\
\hline $\mathrm{C} 9-\mathrm{C} 10-\mathrm{H} 10$ & 119.4 & $\mathrm{C} 8-\mathrm{N} 1-\mathrm{H} 1$ & $115.9(13)$ \\
\hline $\mathrm{O} 2-\mathrm{C} 11-\mathrm{C} 10$ & $115.23(15)$ & $\mathrm{C} 18-\mathrm{N} 2-\mathrm{C} 19$ & $116.17(17)$ \\
\hline $\mathrm{O} 2-\mathrm{C} 11-\mathrm{C} 12$ & $125.25(16)$ & $\mathrm{C} 11-\mathrm{O} 2-\mathrm{C} 14$ & $118.83(14)$ \\
\hline $\mathrm{C} 10-\mathrm{C} 11-\mathrm{C} 12$ & $119.49(16)$ & & \\
\hline
\end{tabular}




\begin{tabular}{|c|c|c|c|}
\hline $\mathrm{C} 6-\mathrm{C} 1-\mathrm{C} 2-\mathrm{F} 1$ & $178.43(17)$ & $\mathrm{C} 10-\mathrm{C} 11-\mathrm{C} 12-\mathrm{C} 13$ & $0.3(3)$ \\
\hline $\mathrm{C} 7-\mathrm{C} 1-\mathrm{C} 2-\mathrm{F} 1$ & $6.5(3)$ & $\mathrm{C} 9-\mathrm{C} 8-\mathrm{C} 13-\mathrm{C} 12$ & $0.6(3)$ \\
\hline $\mathrm{C} 6-\mathrm{C} 1-\mathrm{C} 2-\mathrm{C} 3$ & $0.0(3)$ & $\mathrm{N} 1-\mathrm{C} 8-\mathrm{C} 13-\mathrm{C} 12$ & $-179.67(16)$ \\
\hline $\mathrm{C} 7-\mathrm{C} 1-\mathrm{C} 2-\mathrm{C} 3$ & $-171.90(18)$ & $\mathrm{C} 11-\mathrm{C} 12-\mathrm{C} 13-\mathrm{C} 8$ & $-0.6(3)$ \\
\hline $\mathrm{F} 1-\mathrm{C} 2-\mathrm{C} 3-\mathrm{C} 4$ & $-178.15(19)$ & $\mathrm{O} 2-\mathrm{C} 14-\mathrm{C} 15-\mathrm{C} 19$ & $123.96(19)$ \\
\hline $\mathrm{C} 1-\mathrm{C} 2-\mathrm{C} 3-\mathrm{C} 4$ & $0.2(3)$ & $\mathrm{O} 2-\mathrm{C} 14-\mathrm{C} 15-\mathrm{C} 16$ & $-56.7(2)$ \\
\hline $\mathrm{C} 2-\mathrm{C} 3-\mathrm{C} 4-\mathrm{C} 5$ & $-0.4(3)$ & $\mathrm{C} 19-\mathrm{C} 15-\mathrm{C} 16-\mathrm{C} 17$ & $-0.8(3)$ \\
\hline $\mathrm{C} 3-\mathrm{C} 4-\mathrm{C} 5-\mathrm{C} 6$ & $0.3(4)$ & $\mathrm{C} 14-\mathrm{C} 15-\mathrm{C} 16-\mathrm{C} 17$ & $179.80(17)$ \\
\hline $\mathrm{C} 2-\mathrm{C} 1-\mathrm{C} 6-\mathrm{F} 2$ & $-179.11(18)$ & $\mathrm{C} 15-\mathrm{C} 16-\mathrm{C} 17-\mathrm{C} 18$ & $0.3(3)$ \\
\hline $\mathrm{C} 7-\mathrm{C} 1-\mathrm{C} 6-\mathrm{F} 2$ & $-7.1(3)$ & $\mathrm{C} 16-\mathrm{C} 17-\mathrm{C} 18-\mathrm{N} 2$ & $0.6(3)$ \\
\hline $\mathrm{C} 2-\mathrm{C} 1-\mathrm{C} 6-\mathrm{C} 5$ & $-0.1(3)$ & $\mathrm{C} 16-\mathrm{C} 17-\mathrm{C} 18-\mathrm{Cl} 1$ & $179.99(14)$ \\
\hline $\mathrm{C} 7-\mathrm{C} 1-\mathrm{C} 6-\mathrm{C} 5$ & $171.9(2)$ & $\mathrm{C} 16-\mathrm{C} 15-\mathrm{C} 19-\mathrm{N} 2$ & $0.7(3)$ \\
\hline $\mathrm{C} 4-\mathrm{C} 5-\mathrm{C} 6-\mathrm{F} 2$ & $178.9(2)$ & $\mathrm{C} 14-\mathrm{C} 15-\mathrm{C} 19-\mathrm{N} 2$ & $-179.97(17)$ \\
\hline $\mathrm{C} 4-\mathrm{C} 5-\mathrm{C} 6-\mathrm{C} 1$ & $0.0(4)$ & $\mathrm{O} 1-\mathrm{C} 7-\mathrm{N} 1-\mathrm{C} 8$ & $-1.5(3)$ \\
\hline $\mathrm{C} 6-\mathrm{C} 1-\mathrm{C} 7-\mathrm{O} 1$ & $-77.8(3)$ & $\mathrm{C} 1-\mathrm{C} 7-\mathrm{N} 1-\mathrm{C} 8$ & $178.76(15)$ \\
\hline $\mathrm{C} 2-\mathrm{C} 1-\mathrm{C} 7-\mathrm{O} 1$ & $93.7(2)$ & $\mathrm{C} 13-\mathrm{C} 8-\mathrm{N} 1-\mathrm{C} 7$ & $-170.21(16)$ \\
\hline $\mathrm{C} 6-\mathrm{C} 1-\mathrm{C} 7-\mathrm{N} 1$ & $102.0(2)$ & $\mathrm{C} 9-\mathrm{C} 8-\mathrm{N} 1-\mathrm{C} 7$ & $9.5(3)$ \\
\hline $\mathrm{C} 2-\mathrm{C} 1-\mathrm{C} 7-\mathrm{N} 1$ & $-86.6(2)$ & $\mathrm{C} 17-\mathrm{C} 18-\mathrm{N} 2-\mathrm{C} 19$ & $-0.7(3)$ \\
\hline $\mathrm{C} 13-\mathrm{C} 8-\mathrm{C} 9-\mathrm{C} 10$ & $-0.3(3)$ & $\mathrm{C} 11-\mathrm{C} 18-\mathrm{N} 2-\mathrm{C} 19$ & $179.84(14)$ \\
\hline $\mathrm{N} 1-\mathrm{C} 8-\mathrm{C} 9-\mathrm{C} 10$ & $-179.98(16)$ & $\mathrm{C} 15-\mathrm{C} 19-\mathrm{N} 2-\mathrm{C} 18$ & $0.1(3)$ \\
\hline $\mathrm{C} 8-\mathrm{C} 9-\mathrm{C} 10-\mathrm{C} 11$ & $0.0(3)$ & $\mathrm{C} 10-\mathrm{C} 11-\mathrm{O} 2-\mathrm{C} 14$ & $172.31(14)$ \\
\hline $\mathrm{C} 9-\mathrm{C} 10-\mathrm{C} 11-\mathrm{O} 2$ & $178.18(16)$ & $\mathrm{C} 12-\mathrm{C} 11-\mathrm{O} 2-\mathrm{C} 14$ & $-9.6(2)$ \\
\hline $\mathrm{C} 9-\mathrm{C} 10-\mathrm{C} 11-\mathrm{C} 12$ & $0.0(3)$ & $\mathrm{C} 15-\mathrm{C} 14-\mathrm{O} 2-\mathrm{C} 11$ & $-78.49(19)$ \\
\hline $\mathrm{O} 2-\mathrm{C} 11-\mathrm{C} 12-\mathrm{C} 13$ & $-177.66(16)$ & & \\
\hline
\end{tabular}

Hydrogen-bond geometry $\left(A,{ }^{\circ}\right)$

$\mathrm{Cg} 2$ is the centroid of the $\mathrm{C} 1-\mathrm{C} 6$ benzene ring.

\begin{tabular}{lllll}
\hline$D-\mathrm{H} \cdots A$ & $D-\mathrm{H}$ & $\mathrm{H} \cdots A$ & $D \cdots A$ & $D-\mathrm{H} \cdots A$ \\
\hline $\mathrm{C} 9-\mathrm{H} 9 \cdots \mathrm{O} 1$ & 0.93 & 2.27 & $2.863(2)$ & 121 \\
$\mathrm{~N} 1-\mathrm{H} 1 \cdots \mathrm{N} 2^{\mathrm{i}}$ & $0.88(2)$ & $2.24(2)$ & $3.109(2)$ & $170.6(18)$ \\
$\mathrm{C} 19-\mathrm{H} 19 \cdots \mathrm{F} 2^{\mathrm{ii}}$ & 0.93 & 2.54 & $3.309(2)$ & 140 \\
$\mathrm{C} 14-\mathrm{H} 14 B \cdots \mathrm{O} 1^{\mathrm{ii}}$ & 0.97 & 2.42 & $3.344(3)$ & 160 \\
$\mathrm{C} 16-\mathrm{H} 16 \cdots C g 2^{\mathrm{iii}}$ & 0.93 & 2.99 & $3.912(2)$ & 173 \\
\hline
\end{tabular}

Symmetry codes: (i) $-x,-y+1,-z$; (ii) $x-1, y, z$; (iii) $x-1, y, z+1$. 